\title{
Optically Active Seleninamides: Isolation, Absolute Configuration, and
}

\section{Racemization Mechanism}

\author{
Yusuke Nakashima, Toshio Shimizu, ${ }^{*}$ Kazunori Hirabayashi, and Nobumasa Kamigata ${ }^{*}$ \\ Department of Chemistry, Graduate School of Science, Tokyo Metropolitan University \\ Minami-ohsawa, Hachioji, Tokyo 192-0397, Japan \\ kamigata-nobumasa@c.metro-u.ac.jp
}

Table of Contents

Page

Compound characterization data for selenenamides,

1b, 1d, 3b, 2b, and $(S)-(-)-3 \mathbf{b}$

S2

${ }^{1} \mathrm{H}$ and ${ }^{13} \mathrm{C}$ NMR spectra of $\mathbf{1 b}, \mathbf{1 c}, \mathbf{1 d}$, and $\mathbf{3 a}$

S6

Cartesian coordinates and computed total energies

S14 
2-\{1-Adamantyl\}-1,2-benzisoselenazol-3(2H)-one. $\quad$ Yield $56 \%$; $\mathrm{mp}$ : 194-196 ${ }^{\circ} \mathrm{C}$ (yellow needles from $\mathrm{CDCl}_{3}$.); ${ }^{1} \mathrm{H} \mathrm{NMR}\left(500 \mathrm{MHz}, \mathrm{CDCl}_{3}\right) \delta 1.73(3 \mathrm{H}, \mathrm{d}, J$ $=12.5 \mathrm{~Hz}), 1.80(3 \mathrm{H}, \mathrm{d}, J=12.5 \mathrm{~Hz}), 2.18(3 \mathrm{H}, \mathrm{s}), 2.42(6 \mathrm{H}, \mathrm{s}), 7.39(1 \mathrm{H}, \mathrm{dd}, J=7.95$, $7.40 \mathrm{~Hz}), 7.54(1 \mathrm{H}, \mathrm{dd}, J=7.95,7.40 \mathrm{~Hz}), 7.58(1 \mathrm{H}, \mathrm{d}, J=7.95 \mathrm{~Hz}), 7.95(1 \mathrm{H}, \mathrm{d}, J=$ $7.95 \mathrm{~Hz}) ;{ }^{13} \mathrm{C}$ NMR $\left(125 \mathrm{MHz}, \mathrm{CDCl}_{3}\right) \delta 30.0,36.1,41.4,59.8,123.2,125.7,128.1$, 130.3, 131.2, 137.4, 160.5; ${ }^{77} \mathrm{Se}$ NMR (95 MHz, CDCl 3 ) $\delta 851 ; \mathrm{MS}(\mathrm{EI}, 70 \mathrm{eV}) \mathrm{m} / \mathrm{z} 333$ $\left(\mathrm{M}^{+},{ }^{80} \mathrm{Se}\right), 331\left(\mathrm{M}^{+},{ }^{78} \mathrm{Se}\right), 135$; IR (KBr) 2906, $1599(\mathrm{C}=\mathrm{O}), 1445,1074,731 \mathrm{~cm}^{-1}$. Anal. Calcd for $\mathrm{C}_{17} \mathrm{H}_{19} \mathrm{NOSe}$ N, 4.22; C, 61.45; H, 5.76. Found: N, 4.17; C, 61.49; H, 5.81 .

2-\{2,4,6-Trimethylphenyl\}-1,2-benzisoselenazol-3(2H)-one. Yield 71\%; mp $181{ }^{\circ} \mathrm{C}$ (colorless prisms from acetone; decomp); ${ }^{1} \mathrm{H}$ NMR $\left(500 \mathrm{MHz}, \mathrm{CDCl}_{3}\right) \delta 2.15$ $(6 \mathrm{H}, \mathrm{s}), 2.31(3 \mathrm{H}, \mathrm{s}), 6.95(2 \mathrm{H}, \mathrm{s}), 7.46(1 \mathrm{H}, \mathrm{dd}, J=7.65,7.40 \mathrm{~Hz}), 7.64(1 \mathrm{H}, \mathrm{dd}, J=$ 7.60, $7.40 \mathrm{~Hz}), 7.69(1 \mathrm{H}, \mathrm{d}, J=7.60 \mathrm{~Hz}), 8.13(1 \mathrm{H}, \mathrm{d}, J=7.65 \mathrm{~Hz}) ;{ }^{13} \mathrm{C}$ NMR $(125 \mathrm{MHz}$, $\left.\mathrm{CDCl}_{3}\right) \delta 18.0,21.1,124.2,126.2,126.7,129.3,129.5,132.3,132.7,137.5,139.0$, 139.1, 166.0; ${ }^{77} \mathrm{Se}$ NMR (95 MHz, $\left.\mathrm{CDCl}_{3}\right) \delta$ 920; MS (EI, $\left.30 \mathrm{eV}\right) \mathrm{m} / \mathrm{z} 317\left(\mathrm{M}^{+},{ }^{80} \mathrm{Se}\right)$, $315\left(\mathrm{M}^{+},{ }^{78} \mathrm{Se}\right), 298,183,119$; IR (KBr) 2914, 1614 (C=O), 1441, 1346, $740 \mathrm{~cm}^{-1}$. Anal. Calcd for $\mathrm{C}_{16} \mathrm{H}_{15}$ NOSe: N, 4.43; C, 60.76; H, 4.78. Found: N, 4.36; C, 60.47; H, 4.93.

2-\{2,4,6-Triisopropyphenyl\}-1,2-benzisoselenazol-3(2H)-one. Yield 88\%; mp 183-184 ${ }^{\circ} \mathrm{C}$ (orange powder from acetone/hexane); ${ }^{1} \mathrm{H} \mathrm{NMR}\left(500 \mathrm{MHz}, \mathrm{CDCl}_{3}\right) \delta 1.19$ $(12 \mathrm{H}, \mathrm{d}, J=6.70 \mathrm{~Hz}), 1.27(6 \mathrm{H}, \mathrm{d}, J=7.05 \mathrm{~Hz}), 2.82-2.85(3 \mathrm{H}, \mathrm{m}), 7.07(2 \mathrm{H}, \mathrm{s}), 7.44$ $(1 \mathrm{H}, \mathrm{dd}, J=7.60,7.26 \mathrm{~Hz}), 7.61(1 \mathrm{H}, \mathrm{dd}, J=7.26,7.95 \mathrm{~Hz}), 7.67(1 \mathrm{H}, \mathrm{d}, J=7.95 \mathrm{~Hz})$, $8.13(1 \mathrm{H}, \mathrm{d}, J=7.60 \mathrm{~Hz}) ;{ }^{13} \mathrm{C} \mathrm{NMR}\left(125 \mathrm{MHz}, \mathrm{CDCl}_{3}\right) \delta 23,89,23.90,24.0,28.6,34.3$, $122.1,124.1,126.1,126.5,129.5,129.9,132.2,139.0,147.8,150.0,166.8 ;{ }^{77}$ Se NMR $\left(95 \mathrm{MHz}, \mathrm{CDCl}_{3}\right) . \delta 952 ; \mathrm{MS}(\mathrm{EI}, 70 \mathrm{eV}) \mathrm{m} / \mathrm{z} 401\left(\mathrm{M}^{+},{ }^{80} \mathrm{Se}\right), 384,358,217,202$; IR (KBr) 2961, $1592(\mathrm{C}=\mathrm{O}), 1444,1338,736 \mathrm{~cm}^{-1}$. Anal. Calcd for $\mathrm{C}_{22} \mathrm{H}_{27} \mathrm{NOSe}$ : N, 3.50; C, 65.99; H, 6.80. Found: N, 3.48; C, 65.89; H, 6.81.

2-\{1-Adamantyl\}-1,2-benzisoselenazol-3(2H)-one 1-Oxide (1b). Yield 92\%; 
mp: $203{ }^{\circ} \mathrm{C}$ (colorless powder; decomp.); ${ }^{1} \mathrm{H} \mathrm{NMR}\left(500 \mathrm{MHz}, \mathrm{CDCl}_{3}\right) \delta 1.74(3 \mathrm{H}, \mathrm{d}, J=$ $12.5 \mathrm{~Hz}), 1.82(3 \mathrm{H}, \mathrm{d}, J=12.5 \mathrm{~Hz}), 2.21(3 \mathrm{H}, \mathrm{s}), 2.42(3 \mathrm{H}, \mathrm{d}, J=10.8 \mathrm{~Hz}), 2.52(3 \mathrm{H}, \mathrm{d}$, $J=10.8 \mathrm{~Hz}), 7.70-7.75(2 \mathrm{H}, \mathrm{m}), 7.82(1 \mathrm{H}, \mathrm{d}, J=7.00 \mathrm{~Hz}), 7.95(1 \mathrm{H}, \mathrm{d}, J=7.00 \mathrm{~Hz}) ;{ }^{13} \mathrm{C}$ NMR $\left(125 \mathrm{MHz}, \mathrm{CDCl}_{3}\right) \delta 30.1,36.0,42.6,60.8,125.7,127.9,132.6,132.7,133.7$, 143.6, 167.9; ${ }^{77} \mathrm{Se}$ NMR (95 MHz, $\left.\mathrm{CDCl}_{3}\right) \delta$ 1096; MS (EI, $\left.70 \mathrm{eV}\right) \mathrm{m} / \mathrm{z} 349\left(\mathrm{M}^{+},{ }^{80} \mathrm{Se}\right)$, $333\left(\mathrm{M}^{+},{ }^{80} \mathrm{Se}\right), 135$; IR (KBr) 2907, 1635 (C=O), 1458, 809 (Se=O), $737 \mathrm{~cm}^{-1} \square \mathrm{UV}$ (hexane/2-propanol=3/1): $\lambda_{\max } 273.5$ (sh, $\left.\varepsilon 3.07 \times 10^{3}\right), 231.5$ (sh, $\varepsilon 1.18 \times 10^{4}$ ), $201.5(\varepsilon$ $\left.3.52 \times 10^{4}\right) \mathrm{nm}$; HRMS $(70 \mathrm{eV}) \mathrm{m} / \mathrm{z} 349.0570\left(\mathrm{C}_{17} \mathrm{H}_{19} \mathrm{NO}_{2}{ }^{80}\right.$ Se requires 349.0581).

2-\{2,4,6-Triisopropyphenyl\}-1,2-benzisoselenazol-3(2H)-one 1-Oxide (1d). Yield 73\%; mp $221{ }^{\circ} \mathrm{C}$ (colorless powder from dichloromethane/hexane; decomp); ${ }^{1} \mathrm{H}$ NMR (500 MHz, $\left.\mathrm{CDCl}_{3}\right) \delta 1.16(6 \mathrm{H}, \mathrm{d}, J=6.92 \mathrm{~Hz}), 1.22(3 \mathrm{H}, \mathrm{d}, J=6.92 \mathrm{~Hz}), 1.24(3 \mathrm{H}$, $\mathrm{d}, J=6.92 \mathrm{~Hz}), 1.29(6 \mathrm{H}, \mathrm{d}, J=6.80 \mathrm{~Hz}), 2.75(1 \mathrm{H}, \operatorname{sep}, J=6.92 \mathrm{~Hz}), 2.94(1 \mathrm{H}, \operatorname{sep}, J=$ $6.80 \mathrm{~Hz}), 3.19(1 \mathrm{H}, \mathrm{sep}, J=6.92 \mathrm{~Hz}), 7.09(1 \mathrm{H}, \mathrm{s}), 7.16(1 \mathrm{H}, \mathrm{s}), 7.81(1 \mathrm{H}, \mathrm{dd}, J=7.00$, $7.54 \mathrm{~Hz}), 7.84(1 \mathrm{H}, \mathrm{dd}, J=7.30,7.54 \mathrm{~Hz}), 7.98(1 \mathrm{H}, \mathrm{d}, J=7.30 \mathrm{~Hz}), 8.13(1 \mathrm{H}, \mathrm{d}, J=$ $7.00 \mathrm{~Hz}) ;{ }^{13} \mathrm{C} \mathrm{NMR}\left(125 \mathrm{MHz}, \mathrm{CDCl}_{3}\right) \delta 23.89,23.91,24.0,24.3,24.6,24.7,29.2,29.5$, 34.3, 122.0, 122.7, 126.4, 127.1, 129.0, 129.8, 133.1, 134.5, 145.8, 146.4, 149.6, 150.4, 167.7; ${ }^{77} \mathrm{Se}$ NMR (95 MHz, $\left.\mathrm{CDCl}_{3}\right) . \delta 1052$; MS (EI, $\left.30 \mathrm{eV}\right) \mathrm{m} / \mathrm{z} 417\left(\mathrm{M}^{+},{ }^{80} \mathrm{Se}\right), 401$ $\left(\mathrm{M}^{+}-\mathrm{O},{ }^{80} \mathrm{Se}\right), 384,358,217,202$; IR (KBr) 2963, 1695 (C=O), 1321, 1105, $802(\mathrm{Se}=\mathrm{O})$, $744 \mathrm{~cm}^{-1}$; UV (2-propanol) $\lambda_{\max } 275$ (sh, $\left.\varepsilon 4.81 \times 10^{3}\right), 238\left(\mathrm{sh}, \varepsilon 1.40 \times 10^{4}\right), 201(\varepsilon$ $\left.6.21 \times 10^{4}\right) \mathrm{nm}$; UV (hexane/2-propanol = 3/1) $\lambda_{\max } 274\left(\mathrm{sh}, \varepsilon 2.34 \times 10^{3}\right), 238(\mathrm{sh}$, $\left.\varepsilon 7.92 \times 10^{3}\right), 202\left(\varepsilon 3.80 \times 10^{4}\right) \mathrm{nm}$; HRMS $(30 \mathrm{eV}) \mathrm{m} / \mathrm{z} 417.1192\left(\mathrm{C}_{22} \mathrm{H}_{27} \mathrm{NO}_{2}{ }^{80} \mathrm{Se}\right.$ requires 417.1207).

2-\{2,4,6-Trimethylphenyl\}-1,2-benzisothiazol-3(2H)-one. To a dichloromethane $(10 \mathrm{ml})$ solution of 2,4,6-trimethylaniline $(270 \mathrm{mg}, 2.00 \mathrm{mmol})$ and triethylamine $(0.28 \mathrm{ml}, 1.0 \mathrm{mmol})$ was slowly added a dichloromethane $(20 \mathrm{ml})$ solution of 2,2'-dithiodibenzoyl chloride $(343 \mathrm{mg}, 1.00 \mathrm{mmol})$ over $15 \mathrm{~min}$. After stirring the mixture for $3 \mathrm{~h}$, the mixture was washed with $1 \mathrm{M} \mathrm{HCl}(50 \mathrm{ml})$, water $(50 \mathrm{ml})$, and brine 
(50 ml), and dried over anhydrous sodium sulfate. The solution was concentrated under reduced pressure and purification by silica gel column chromatography (hexane/acetone) afforded the product $\left(218 \mathrm{mg}\right.$ ). Yield $81 \%$; mp $135^{\circ} \mathrm{C}$ (pale orange needles from dichloromethane/hexane; decomp); ${ }^{1} \mathrm{H}$ NMR (400 MHz, $\left.\mathrm{CDCl}_{3}\right) \delta 2.03$ $(6 \mathrm{H}, \mathrm{s}), 2.19(3 \mathrm{H}, \mathrm{s}), 6.85(2 \mathrm{H}, \mathrm{s}), 7.30(1 \mathrm{H}, \mathrm{dd}, J=7.80,6.80 \mathrm{~Hz}), 7.45(1 \mathrm{H}, \mathrm{d}, J=8.08$ $\mathrm{Hz}), 7.50(1 \mathrm{H}, \mathrm{dd}, J=8.08,6.80 \mathrm{~Hz}), 8.00(1 \mathrm{H}, \mathrm{d}, J=7.80 \mathrm{~Hz}) ;{ }^{13} \mathrm{C} \mathrm{NMR}(100 \mathrm{MHz}$, $\left.\mathrm{CDCl}_{3}\right) \delta 17.8,21.1,120.3,123.8,135.1,126.8,129.0,130.4,131.8,137.4,139.2$, 141.1, 164.1; MS (EI, 30 eV) m/z $269\left(\mathrm{M}^{+}\right), 252$, 120, 91; IR (KBr) 3068, 2918, 1646 $(\mathrm{C}=\mathrm{O}), 1444,1331,735,674,557 \mathrm{~cm}^{-1}$. Anal. Calcd for $\mathrm{C}_{16} \mathrm{H}_{15} \mathrm{NOS}: \mathrm{N}, 5.20 ; \mathrm{C}, 71.34$; H, 5.61. Found: N, 4.82; C, 71.23; H, 5.61.

2-\{2,4,6-Trimethylphenyl\}-1,2-benzisothiazol-3(2H)-one 1-Oxide (2b). Ozone was bubbled into a dichloromethane (30 ml) solution of the 2-\{2,4,6-trimethylphenyl $\}$ 1,2-benzisothiazol-3(2H)-one $(269 \mathrm{mg}, 1.00 \mathrm{mmol})$ at $-20{ }^{\circ} \mathrm{C}$ for $6 \mathrm{~min}$. Removal of the solvent under reduced pressure afforded $\mathbf{2 b}$ in quantitative yield. Mp $145-146{ }^{\circ} \mathrm{C}$ (colorless blocks from dichloromethane/hexane); ${ }^{1} \mathrm{H}$ NMR $\left(500 \mathrm{MHz}, \mathrm{CDCl}_{3}\right) \delta 2.10$ $(3 \mathrm{H}, \mathrm{s}), 2.31(3 \mathrm{H}, \mathrm{s}), 2.33(3 \mathrm{H}, \mathrm{s}), 7.00(1 \mathrm{H}, \mathrm{s}), 7.03(1 \mathrm{H}, \mathrm{s}), 7.80(1 \mathrm{H}, \mathrm{dd}, J=7.40,7.48$ Hz), $7.85(1 \mathrm{H}, \mathrm{dd}, J=7.65,7.48 \mathrm{~Hz}), 7.96(1 \mathrm{H}, \mathrm{d}, J=7.65 \mathrm{~Hz}), 8.08(1 \mathrm{H}, \mathrm{d}, J=7.40$ $\mathrm{Hz}) ;{ }^{13} \mathrm{C} \mathrm{NMR}\left(125 \mathrm{MHz}, \mathrm{CDCl}_{3}\right) \delta 18.1,18.5,21.0,125.2,126.5,127.5,128.0,129.3$, 129.6, 133.2, 134.4, 136.7, 139.3, 139.7, 146.2, 164.5; MS (EI, 70 eV) m/z $284\left(\mathrm{M}^{+}-1\right)$, 268, 251, 220, 149, 133; IR (KBr) 2915, 1713 (C=O), 1461, 1317, 1292, 1256, 1116, $1085(\mathrm{~S}=\mathrm{O}), 749,680,555 \mathrm{~cm}^{-1}$; UV (2-propanol) $\lambda_{\max } 273$ (ع 3.28x 10), $201(\varepsilon 6.05 \mathrm{x}$ $10^{4}$ ) nm; Anal. Calcd for $\mathrm{C}_{16} \mathrm{H}_{15} \mathrm{NO}_{2} \mathrm{~S}: \mathrm{N}, 4.91 ; \mathrm{C}, 67.34 ; \mathrm{H}, 5.30$. Found: N, 5.09; C, $67.05 ; \mathrm{H}, 5.21$.

Methyl 2-N-(2,4,6-trimethylphenyl)aminocarbonylphenyl sulfoxide $\quad(3 \mathrm{~b}$, racemic). $84 \%$; mp $158{ }^{\circ} \mathrm{C}$ (colorless powder from acetone/hexane; decomp); ${ }^{1} \mathrm{H}$ NMR (500 MHz, $\left.\mathrm{CDCl}_{3}\right) \delta 2.23(6 \mathrm{H}, \mathrm{s}), 2.28(3 \mathrm{H}, \mathrm{s}), 2.87(3 \mathrm{H}, \mathrm{s}), 6.93(2 \mathrm{H}, \mathrm{s}), 7.59$ $(1 \mathrm{H}, \mathrm{dd}, J=7.60,8.54 \mathrm{~Hz}), 7.75(1 \mathrm{H}, \mathrm{dd}, J=7.65,8.54 \mathrm{~Hz}), 7.84(1 \mathrm{H}, \mathrm{d}, J=7.65 \mathrm{~Hz})$, 
$7.89(1 \mathrm{H}, \mathrm{s}), 8.24(1 \mathrm{H}, \mathrm{d}, J=7.60 \mathrm{~Hz}) ;{ }^{13} \mathrm{C} \mathrm{NMR}\left(125 \mathrm{MHz}, \mathrm{CDCl}_{3}\right) \delta 18.3,20.9,45.0$, 124.6, 126.31, 126.35, 129.1, 130.4, 131.8, 132.5, 135.2, 137.6, 148.6, 165.1; MS (EI, 70 eV) m/z $301\left(\mathrm{M}^{+}\right), 285$ (M+-O), 270 (M+-O-Me), 167, 151, 135; IR (KBr) 3203 (br, $\mathrm{NH}), 2908,1637(\mathrm{C}=\mathrm{O}), 1523,1314,1076(\mathrm{~S}=\mathrm{O}) \mathrm{cm}^{-1}$; Anal. Calcd for $\mathrm{C}_{17} \mathrm{H}_{19} \mathrm{NO}_{2} \mathrm{~S}: \mathrm{N}$, 4.65; C, 67.74; H, 6.35. Found: N, 4.67; C, 67.53; H, 6.34.

Compound (S)-(-)-3b. $60 \% ; 52 \%$ ee; mp $151{ }^{\circ} \mathrm{C}$ (colorless powder; decomp); $[\alpha]_{\mathrm{D}}^{26}-56$ (c 0.018, ethanol). ${ }^{1} \mathrm{H}$ NMR spectrum was almost same as racemic sample. 


\section{${ }^{1} \mathrm{H}$ and ${ }^{13} \mathrm{C}$ NMR spectra of $1 \mathrm{~b}, 1 \mathrm{c}, 1 \mathrm{~d}$, and $3 \mathrm{a}$}

${ }^{1} \mathrm{H}$ NMR spectrum of $\mathbf{1 b}$

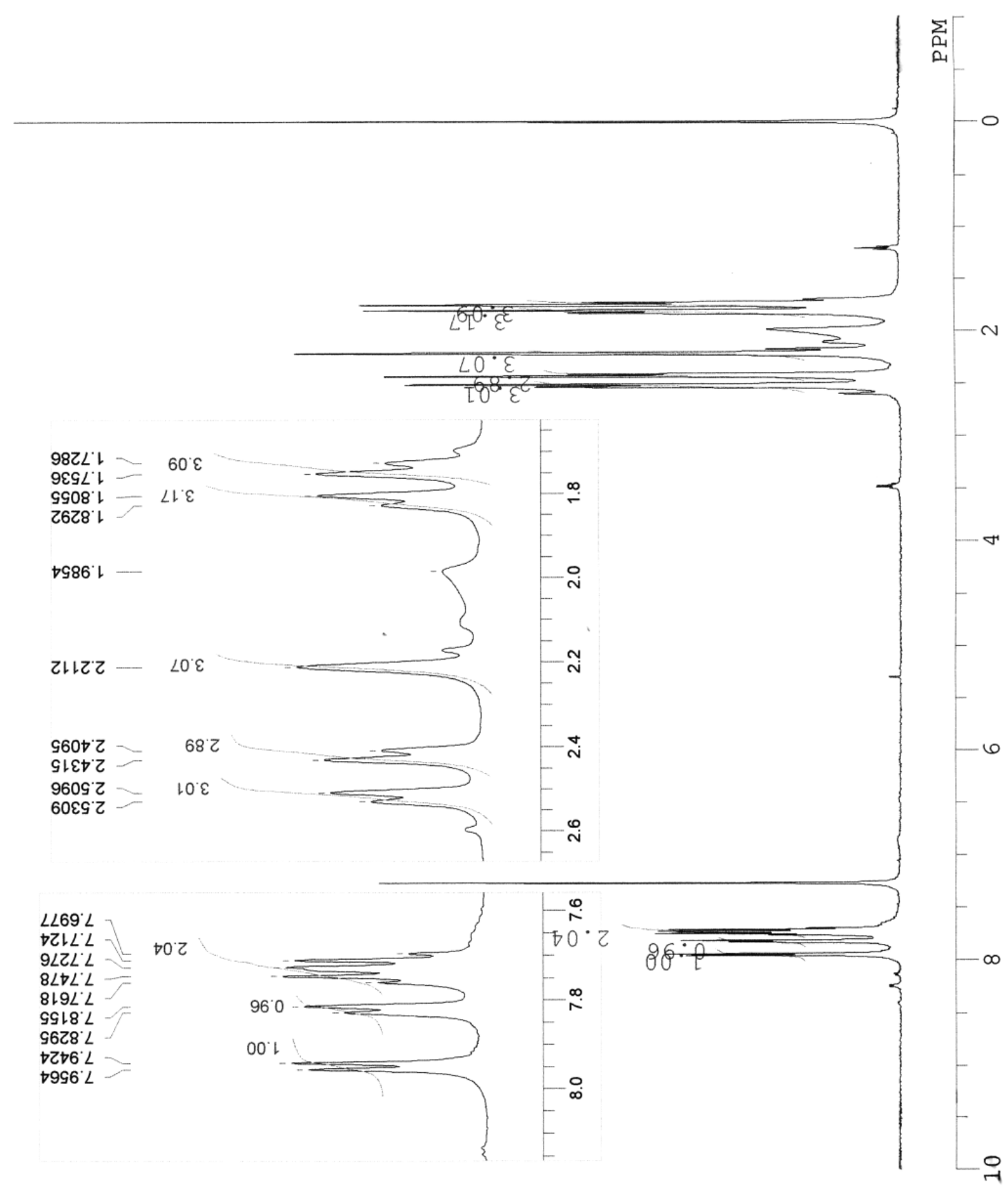


${ }^{13} \mathrm{C}$ NMR spectrum of $\mathbf{1 b}$

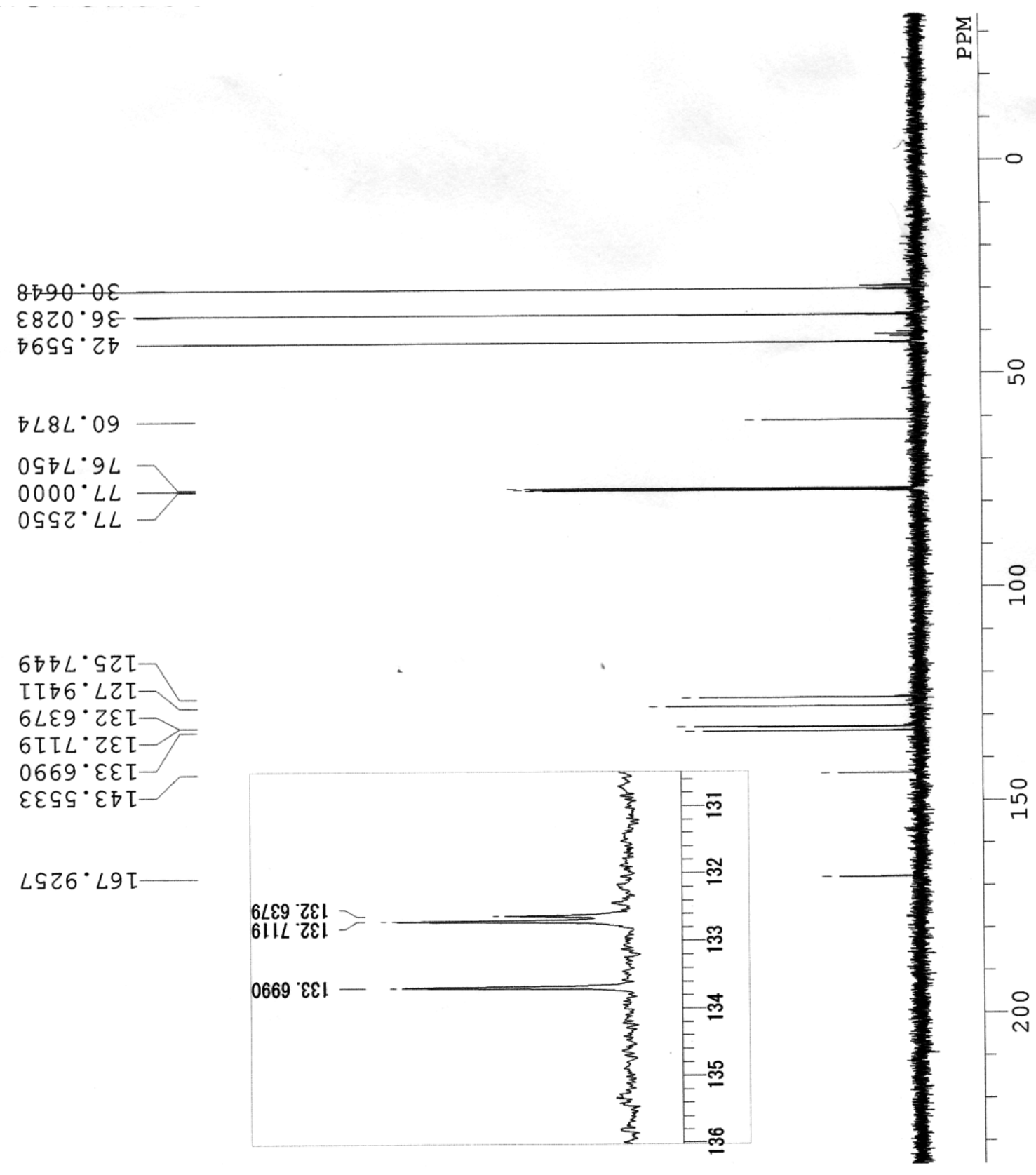


${ }^{1} \mathrm{H}$ NMR spectrum of $\mathbf{1 c}$

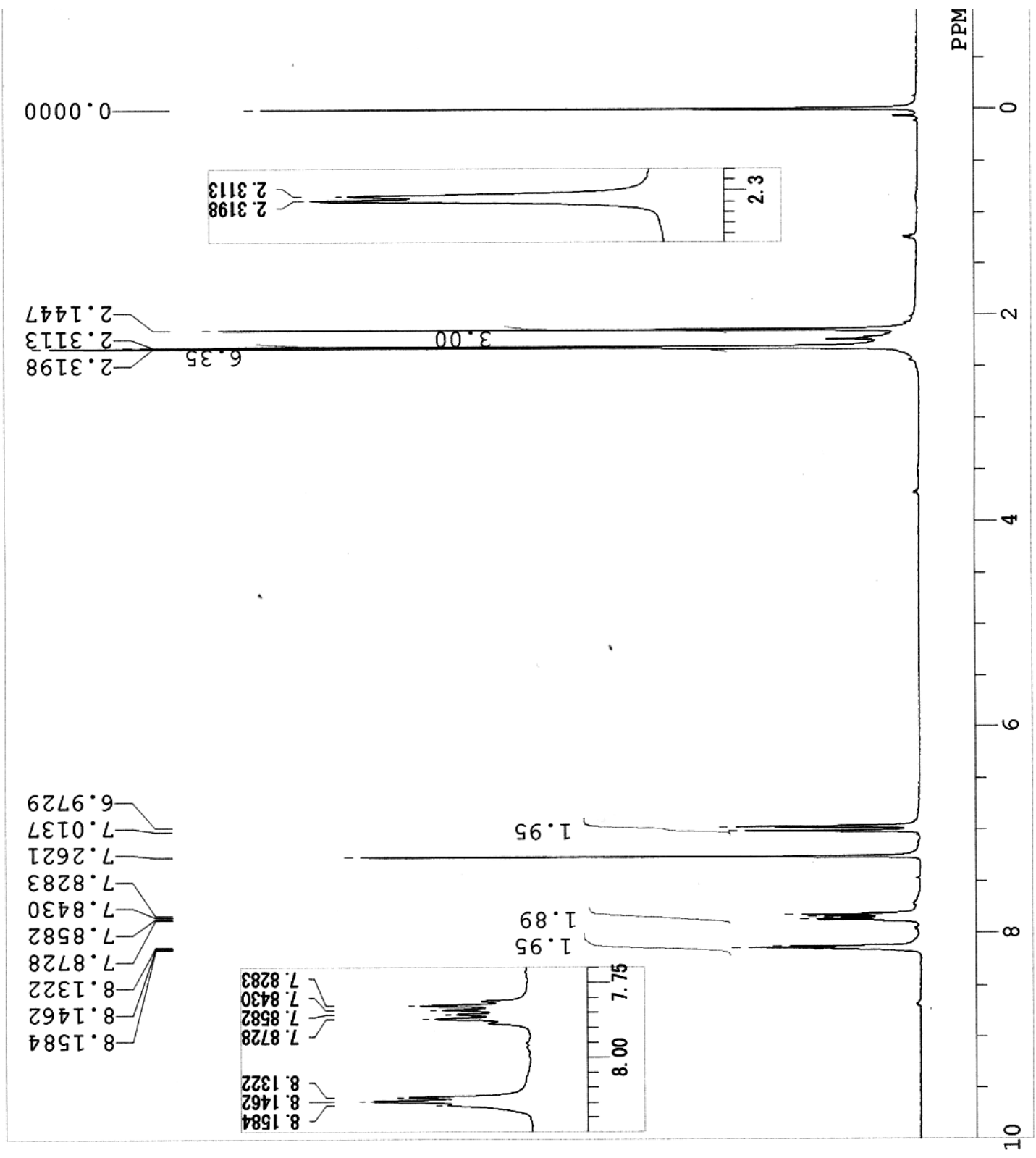


${ }^{13} \mathrm{C}$ NMR spectrum of $\mathbf{1 c}$

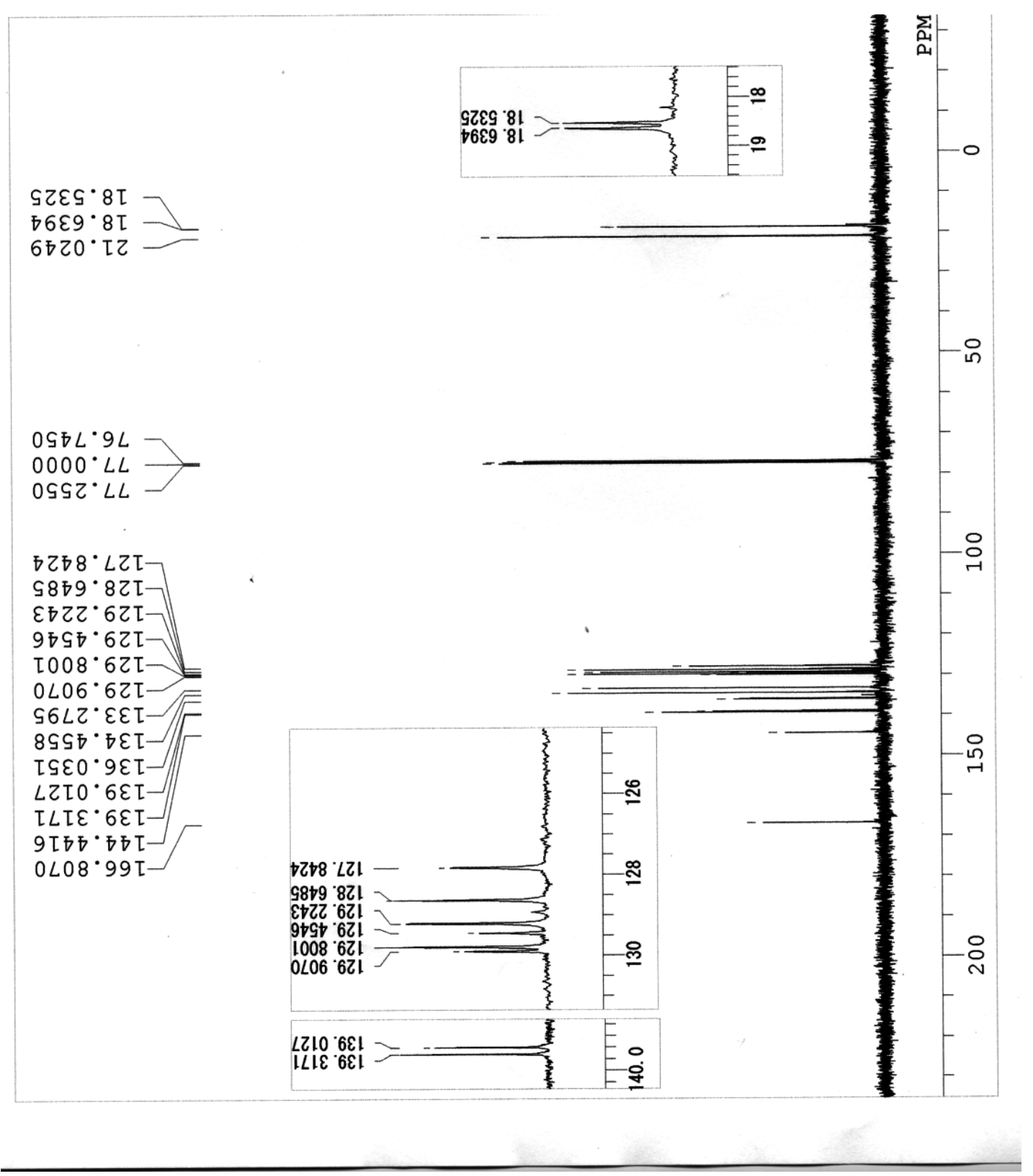


${ }^{1} \mathrm{H}$ NMR spectrum of $\mathbf{1 d}$

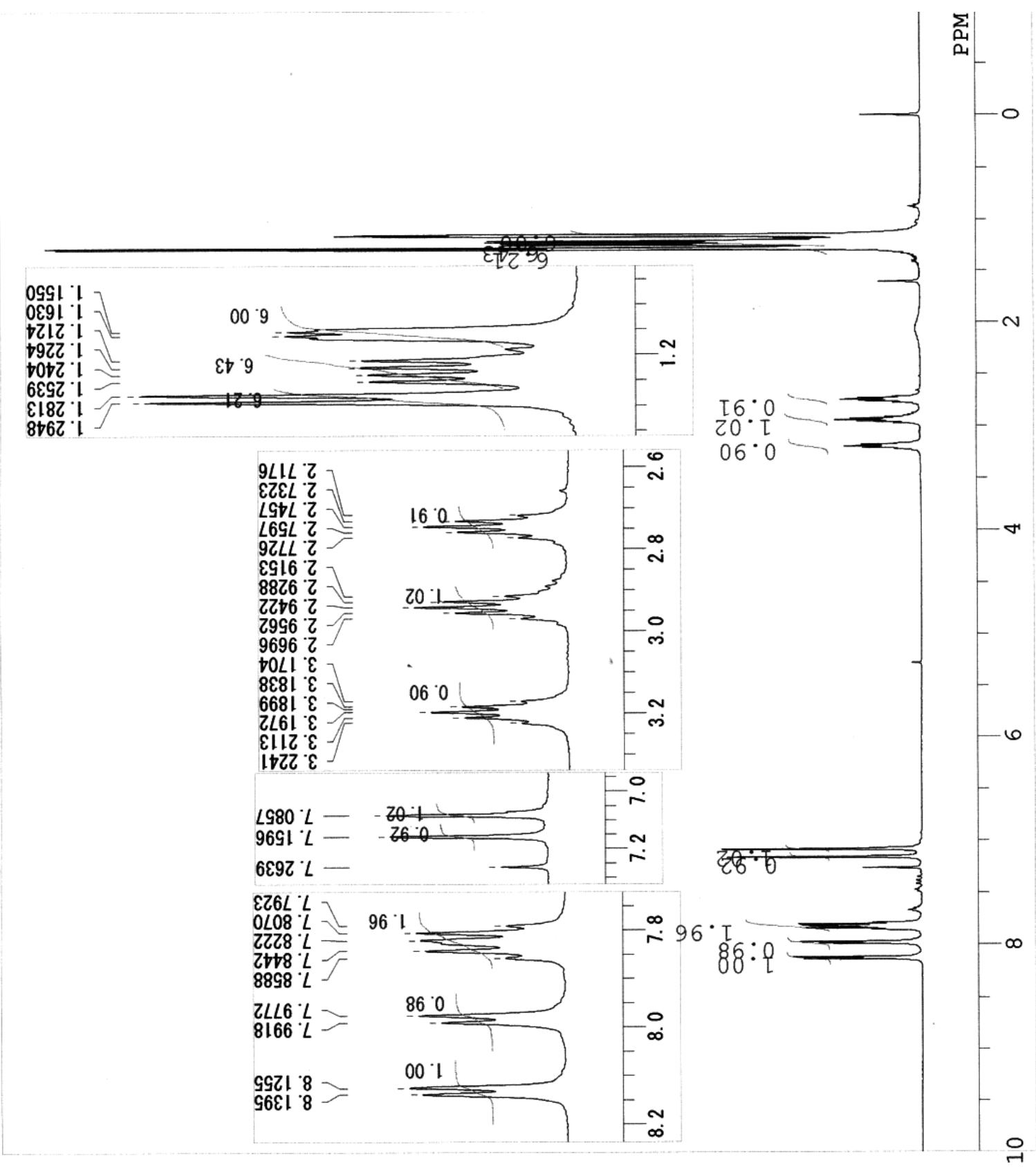


${ }^{13} \mathrm{C}$ NMR spectrum of $\mathbf{1 d}$

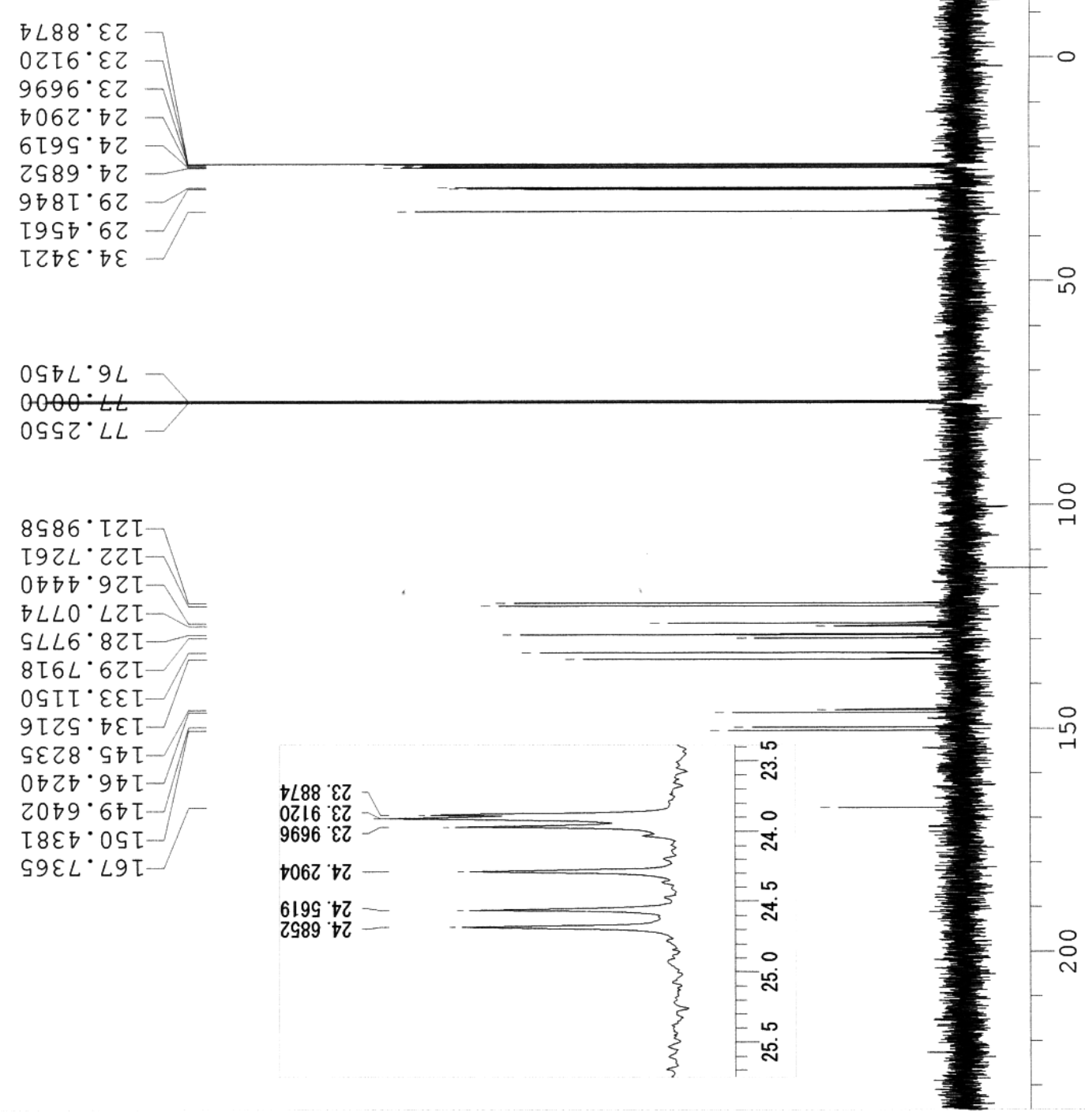


${ }^{1} \mathrm{H}$ NMR spectrum of $\mathbf{3 a}$

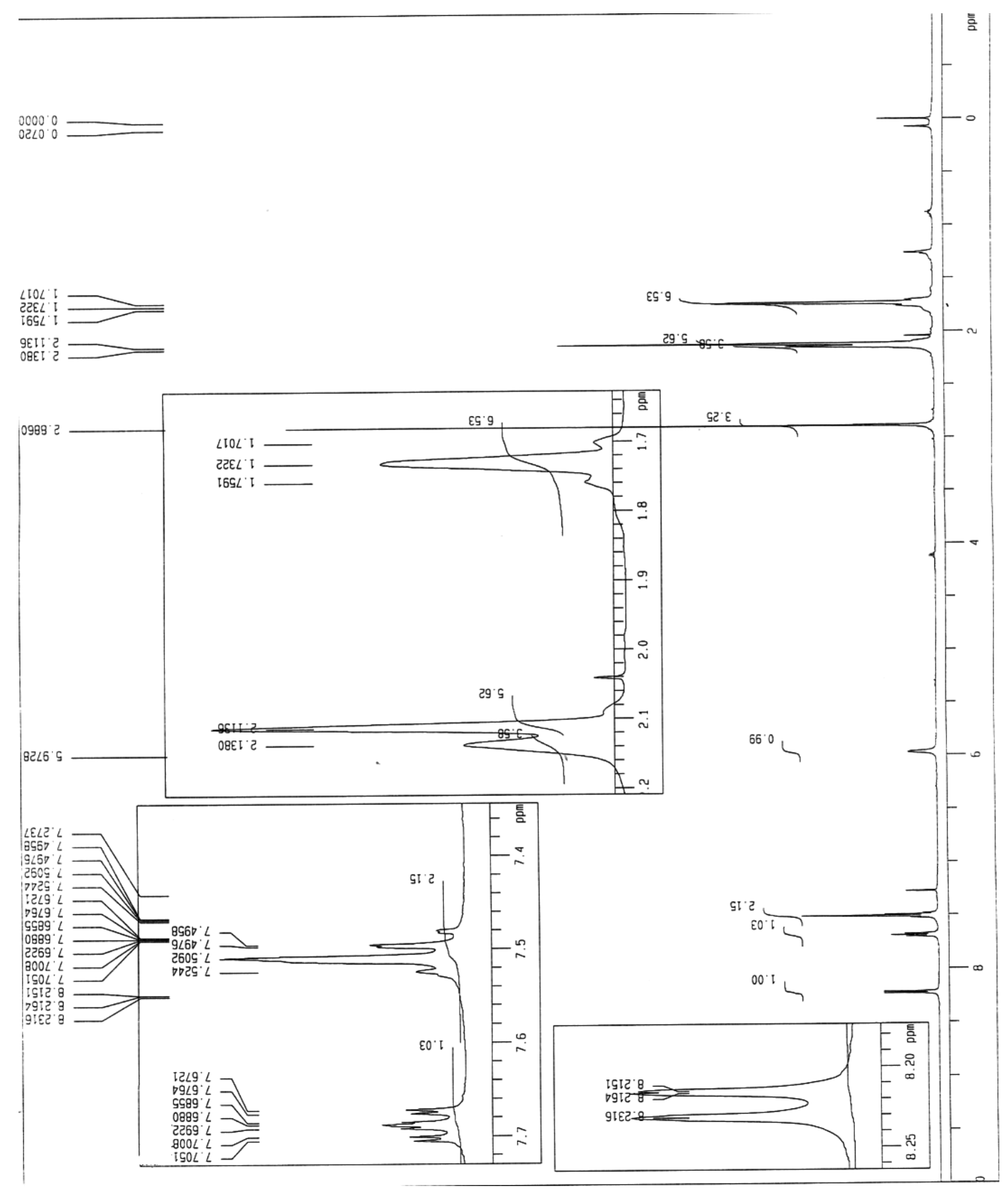


${ }^{13} \mathrm{C}$ NMR spectrum of $\mathbf{3 a}$

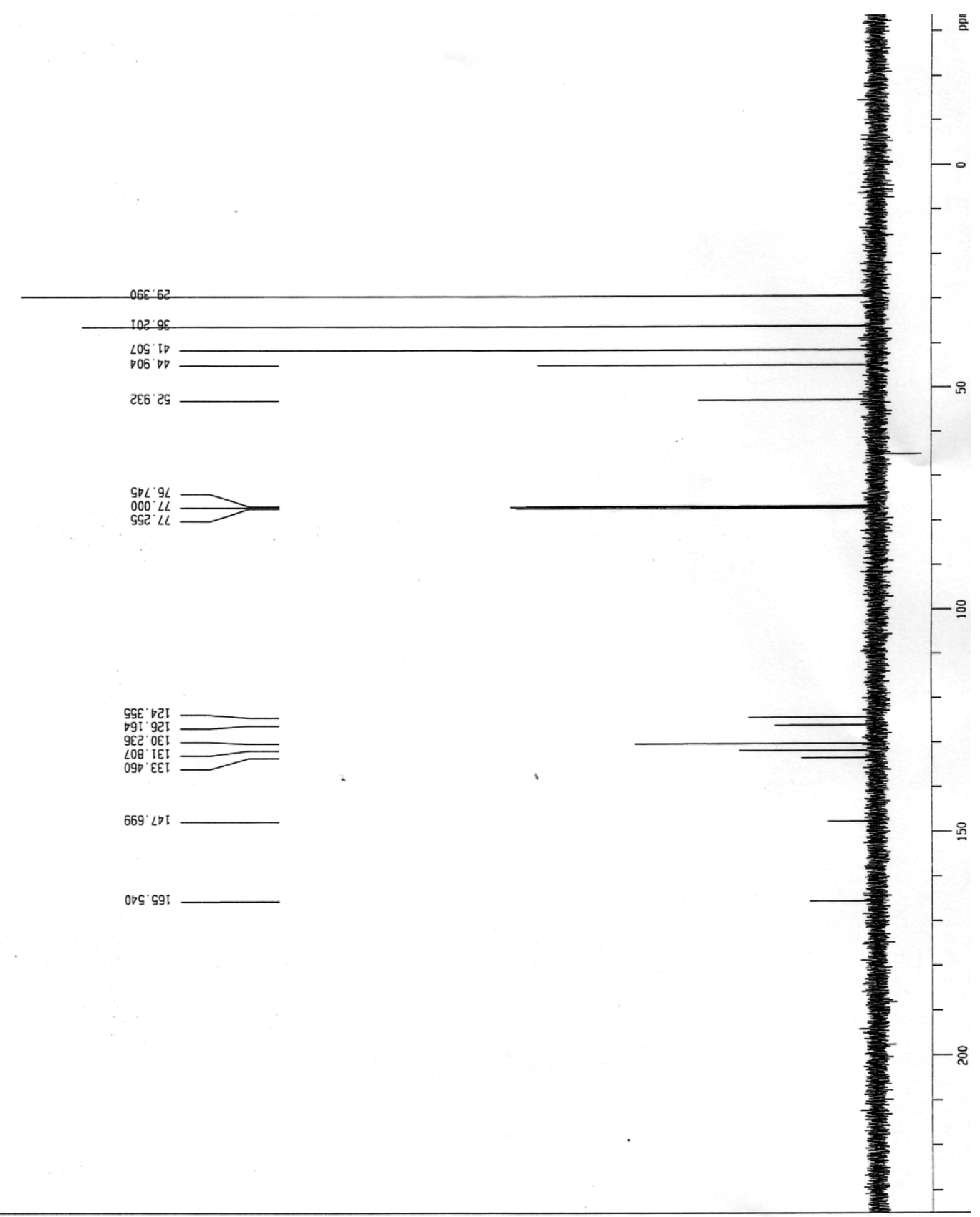




\section{Cartesian Coordinates and Computed Total Energies}

General. Geometries were optimized using the MP2 method with the LANL2DZ basis set. All calculations were performed by using the Gaussian98 program. Sum of electronic and thermal free energies were calculated using scaling factor of 0.9434 at standard state $(298.15 \mathrm{~K}, 1 \mathrm{~atm})$.

\section{Compound 4}

Ground state

Energy $=-521.05243758293$ hartree

Sum of electronic and thermal free energies $=-520.964280$ hartree

Imaginary frequencies $=0$

Cartesian coordinates: Charge $=0$, Multiplicity $=1$

$\begin{array}{lrrr}\mathrm{C} & .806174 & -.567208 & -.149542 \\ \mathrm{C} & 1.876689 & -1.495464 & -.128757 \\ \mathrm{C} & 3.203299 & -.986641 & .007174 \\ \mathrm{C} & 3.433592 & .419496 & .117108 \\ \mathrm{C} & 2.345529 & 1.337761 & .105775 \\ \mathrm{C} & 1.025939 & .830464 & -.033358 \\ \mathrm{C} & -.216437 & 1.697911 & .009638 \\ \mathrm{O} & -.220753 & 2.962283 & .146146 \\ \mathrm{~N} & -1.369643 & .899647 & -.129110 \\ \mathrm{Se} & -1.129824 & -1.042365 & -.291097 \\ \mathrm{O} & -1.512808 & -1.820932 & 1.181686 \\ \mathrm{C} & -2.743150 & 1.464448 & .024023 \\ \mathrm{H} & 1.702547 & -2.574778 & -.188544 \\ \mathrm{H} & 4.049332 & -1.679847 & .032884 \\ \mathrm{H} & 4.456998 & .791738 & .221876 \\ \mathrm{H} & 2.494396 & 2.416173 & .207128 \\ \mathrm{H} & -3.254677 & 1.005889 & .887719 \\ \mathrm{H} & -3.337183 & 1.302466 & -.891700 \\ \mathrm{H} & -2.631220 & 2.545814 & .196697\end{array}$




\section{Transition state for vertex inversion}

Energy $=-520.94284248617$ hartree

Imaginary frequencies $=1$

Cartesian coordinates: Charge $=0$, Multiplicity $=1$

$\begin{array}{llll}\text { C } & .840911 & -.663766 & .001509\end{array}$

$\begin{array}{llll}\mathrm{C} & 1.850326 & -1.658317 & .003060\end{array}$

$\begin{array}{llll}\text { C } & 3.197792 & -1.202500 & .003103\end{array}$

$\begin{array}{llll}\text { C } & 3.502883 & .198179 & .001828\end{array}$

$\begin{array}{llll}\mathrm{C} & 2.466073 & 1.165908 & -.000973\end{array}$

$\begin{array}{llll}\text { C } & 1.103357 & .740309 & -.001873\end{array}$

$\begin{array}{llll}\text { C } & -.060440 & 1.703749 & -.004644\end{array}$

$\begin{array}{llll}\mathrm{O} & .044563 & 2.977795 & -.002104\end{array}$

$\mathrm{N} \quad-1.286582 \quad 1.020134 \quad-.006827$

Se $\quad-1.099738 \quad-.886625 \quad-.001535$

$\begin{array}{lllll}\mathrm{O} & -2.458665 & -2.030850 & -.001104\end{array}$

$\begin{array}{llll}\text { C } & -2.621971 & 1.678576 & .010498\end{array}$

$\mathrm{H} \quad 1.614639 \quad-2.725400 \quad .003515$

$\mathrm{H} \quad 4.007721 \quad-1.937824 \quad .004214$

$\begin{array}{llll}\mathrm{H} & 4.547492 & .521253 & .002058\end{array}$

$\begin{array}{llll}\mathrm{H} & 2.671419 & 2.239548 & -.003150\end{array}$

$\mathrm{H} \quad-3.181503 \quad 1.392129 \quad .915881$

$\begin{array}{llll}\mathrm{H} & -3.198299 & 1.404909 & -.888116\end{array}$

$\begin{array}{llll}\mathrm{H} & -2.425067 & 2.761313 & .016169\end{array}$

\section{Hydrate form (selenurane)}

Sum of electronic and thermal free energies $=-597.076956$ hartree Imaginary frequencies $=0$

Cartesian coordinates: Charge $=0$, Multiplicity $=1$

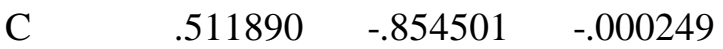

$\begin{array}{llll}\text { C } & 1.598101 & -1.760126 & -.000238\end{array}$

$\begin{array}{llll}\text { C } & 2.916169 & -1.214448 & -.000003\end{array}$

$\begin{array}{llll}\text { C } & 3.120474 & .201066 & -.000002\end{array}$

$\begin{array}{llll}\mathrm{C} & 2.013172 & 1.094535 & -.000390\end{array}$

$\begin{array}{llll}\mathrm{C} & .698325 & .553291 & -.000554\end{array}$

$\begin{array}{llll}\mathrm{C} & -.560535 & 1.396305 & -.000897\end{array}$

$\begin{array}{llll}\mathrm{O} & -.591661 & 2.667769 & -.000996\end{array}$

$\begin{array}{llll}\mathrm{N} & -1.706157 & .576415 & -.001460\end{array}$

$\begin{array}{llll}\mathrm{Se} & -1.416456 & -1.317961 & .000617\end{array}$ 


$\begin{array}{lrrr}\mathrm{O} & -1.445659 & -1.386447 & 1.976687 \\ \mathrm{O} & -1.447767 & -1.390575 & -1.975124 \\ \mathrm{C} & -3.095473 & 1.115117 & .000344 \\ \mathrm{H} & 1.439970 & -2.841804 & -.000229 \\ \mathrm{H} & 3.778691 & -1.887426 & .000188 \\ \mathrm{H} & 4.139323 & .599162 & .000334 \\ \mathrm{H} & 2.141842 & 2.180287 & -.000509 \\ \mathrm{H} & -3.630939 & .785210 & .906303 \\ \mathrm{H} & -3.633227 & .784925 & -.904136 \\ \mathrm{H} & -3.011745 & 2.212208 & .000070 \\ \mathrm{H} & -.907316 & -.754593 & 2.516478 \\ \mathrm{H} & -.911017 & -.758418 & -2.516241\end{array}$

\section{Compound 5}

\section{Ground state}

Energy $=-521.90162010659$ hartree

Sum of electronic and thermal free energies $=-521.811459$ hartree Imaginary frequencies $=0$

Cartesian coordinates: Charge $=0$, Multiplicity $=1$

$\begin{array}{lrrr}\mathrm{C} & .688785 & -.634927 & -.203322 \\ \mathrm{C} & 1.877176 & -1.400220 & -.188903 \\ \mathrm{C} & 3.106838 & -.695539 & -.017713 \\ \mathrm{C} & 3.117750 & .726465 & .132411 \\ \mathrm{C} & 1.903781 & 1.471389 & .126665 \\ \mathrm{C} & .680516 & .771824 & -.047442 \\ \mathrm{C} & -.695244 & 1.401081 & -.006931 \\ \mathrm{O} & -.945631 & 2.634166 & .171470 \\ \mathrm{~N} & -1.664707 & .401441 & -.205836 \\ \mathrm{~S} & -1.048141 & -1.364518 & -.393112 \\ \mathrm{O} & -1.344581 & -2.227055 & .950814 \\ \mathrm{C} & -3.126551 & .627772 & -.044108 \\ \mathrm{H} & 1.862420 & -2.490048 & -.280571 \\ \mathrm{H} & 4.049488 & -1.250187 & .005020 \\ \mathrm{H} & 4.071585 & 1.245759 & .263648 \\ \mathrm{H} & 1.887395 & 2.556506 & .257773 \\ \mathrm{H} & -3.526252 & -.020450 & .754625 \\ \mathrm{H} & -3.660406 & .432745 & -.989314 \\ \mathrm{H} & -3.257632 & 1.683910 & .237248\end{array}$




\section{Hydrate form (sulfurane)}

Sum of electronic and thermal free energies $=-597.903335$ hartree Imaginary frequencies $=0$

Cartesian coordinates: Charge $=0$, Multiplicity $=1$

$\begin{array}{llll}\text { C } & .774661 & -.593466 & .080160\end{array}$

$\begin{array}{llll}\text { C } & 1.959257 & -1.354400 & .145495\end{array}$

$\begin{array}{llll}\text { C } & 3.190036 & -.633882 & .088389\end{array}$

$\begin{array}{llll}\text { C } & 3.202665 & .790809 & -.041698\end{array}$

$\begin{array}{llll}\mathrm{C} & 1.986415 & 1.524915 & -.129911\end{array}$

$\begin{array}{llll}\text { C } & .760158 & .812520 & -.068400\end{array}$

C $\quad-.614130 \quad 1.416408 \quad-.195073$

O $\quad-.899039 \quad 2.646193 \quad-.279962$

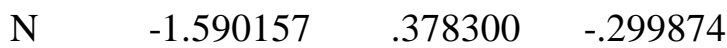

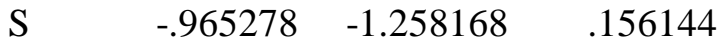

O $\quad-1.067236 \quad-.520170 \quad 2.049402$

$\begin{array}{llll}\mathrm{O} & -.907006 & -1.899387 & -1.714722\end{array}$

C $\quad-3.038838 \quad .643572 \quad-.069066$

$\mathrm{H} \quad 1.942459 \quad-2.443620 \quad .223976$

$\mathrm{H} \quad 4.135946 \quad-1.181190 \quad .139921$

$\begin{array}{llll}\mathrm{H} & 4.159510 & 1.319097 & -.082007\end{array}$

$\begin{array}{llll}\mathrm{H} & 1.971563 & 2.611902 & -.244055\end{array}$

$\begin{array}{llll}\mathrm{H} & -3.276449 & .554113 & 1.004251\end{array}$

$\begin{array}{llll}\mathrm{H} & -3.636052 & -.059269 & -.670447\end{array}$

$\mathrm{H} \quad-3.223558 \quad 1.672485 \quad-.410553$

$\mathrm{H} \quad-1.179312 \quad-1.270566 \quad 2.690230$

$\mathrm{H} \quad-.653665 \quad-1.172313 \quad-2.347610$

Water

Sum of electronic and thermal free energies $=-76.133368$ hartree Imaginary frequencies $=0$

Cartesian coordinates: Charge $=0$, Multiplicity $=1$

$\begin{array}{llll}\mathrm{O} & .000000 & .000000 & .111416 \\ \mathrm{H} & .000000 & .804565 & -.445666 \\ \mathrm{H} & .000000 & -.804565 & -.445666\end{array}$

